\title{
INVESTIGATION OF THE RETRONASAL PERCEPTION OF PALM WINE (ELAEIS GUINEENSIS) AROMA BY APPLICATION OF SENSORY ANALYSIS AND EXHALED ODORANT MEASUREMENT (EXOM)
}

\section{Lasekan $\mathrm{O}^{1,3}$, Buettner $\mathrm{A}^{1,2,4 *}$ and $\mathrm{M}$ Christlbauer ${ }^{1}$}

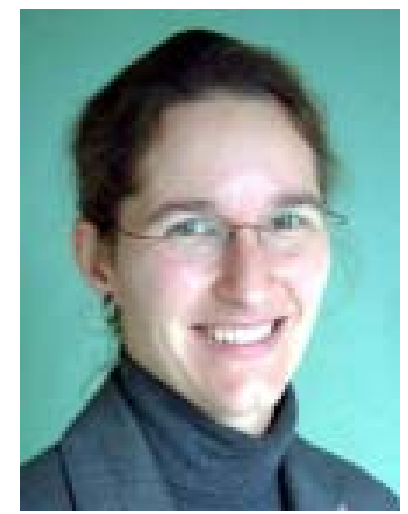

Andrea Buettner

*Corresponding author email: andrea.buettner@1mchemie.uni-erlangen.de

${ }^{1}$ Deutsche Forschungsanstalt fuer Lebensmittelchemie, Lichtenbergstr. 4, D-85748, Garching, Germany

${ }^{2}$ Fraunhofer Institute for Process Engineering and Packaging (IVV), Giggenhauser Str. 35, D-85354 Freising, Germany

${ }^{3}$ Present address: Department of Food Science, University of Agriculture, P.O. Box 2240, Abeokuta Ogun State, Nigeria

${ }^{4}$ Present address: Institute of Pharmacy and Food Chemistry, Friedrich-AlexanderUniversity, Schuhstr. 19, D-91052 Erlangen, Germany 


\section{ABSTRACT}

The headspace profile of palm wine was evaluated by time-resolved sensory analysis showing significant changes of the diverse odour attributes with time after swallowing. Fruity and citrusy were the most intense aroma qualities perceived upon sample introduction into the mouth, while swallowing of the palm wine elicited a more acidic impression followed by citrusy and fruity nuances respectively. After swallowing, panelists described an increase in the nutty and popcorn-like aroma impressions.

Based on these sensory observations, the retronasal aroma perception of palm wine was investigated by application of the modified Exhaled Odorant Measurement (EXOM)-approach. In EXOM analysis, odorants that are exhaled through the nose during food consumption and swallowing are collected and analysed by high resolution gas chromatography-olfactometry and mass spectrometry, respectively. EXOM results revealed an initial 24 odor-active compounds in the 'swallow' breath with 23 of these odorants being identified on the basis of their odor qualities and intensities, as well as chromatographic and mass spectral data. Only 14 compounds were detectable in the exhaled breath $20 \mathrm{~s}$ after swallowing the palm wine and 11 of these were subsequently identified. Generally, the identified odorants belonged to very diverse odorant substance classes such as heteroaromatic compounds, esters, alcohols, carbonyl and thio compounds and many more. Among these, higher persistence intervals in the exhalation breath were obtained for the buttery smelling compounds butan-2,3-dione and 3-hydroxy-butan-2-one (acetoine), 3-isobutyl 2methoxypyrazine with bell pepper-like aroma impression, the malty smelling 2- and 3-methylbutanols, and the coconut-like smelling $\gamma$-dodecalactone. The popcorn-like smelling 2-acetyl 1-pyrroline, the fresh flowery linalool and two unknown compounds with citrusy and buttery aroma impressions were only detectable at $20 \mathrm{~s}$ after swallowing. Dynamic changes were also observed in retronasal sensory evaluations that were attributed to specific palm wine odorants. Accordingly, both sensory and analytical data on retronasal aroma perception of palm wine monitored the dynamic flavour changes during palm wine consumption.

Key words: Retronasal, EXOM, swallow breath, 2-acetyl 1-pyrroline 


\section{INTRODUCTION}

Palm wine is a whitish, effervescent, alcoholic beverage produced by the spontaneous yeast-lactic fermentation of the sugary sap of palm trees. To date, more than 80 volatile compounds have been identified in different palm wine varieties [1,2]. Systematic studies on the odor-active contributors to palm wine aroma were recently reported with identification of those compounds which induce the characteristic alcoholic, malty, and floral-fruity notes of palm wine [3].

Like all liquid foods, palm wine is consumed almost immediately (typically within 2$3 \mathrm{~s}$ of ingestion); a proportion of the flavor-enriched liquid remains in the mouth as a thin film coating the oral cavity [3]. While some food aromas can be perceived for just a short period [4], palm wine aroma lingers for a considerable time after consumption. Prolonged retronasal aroma perception, as it is perceived after complete swallowing of a food, must be induced by persistent odorants which are present in the oral cavity for a certain period of time. That means that the odorants or the respective food matrix are either adsorbed to oral mucosa as a kind of aroma reservoir, and that aroma compounds are released therefrom continuously [5], Another possibility is that odorants are newly generated from less or non-odour active precursor compounds [6]. The adsorption of odorants, as well as the adsorption of food matrix material, has been previously shown to occur [7]. Both processes can be regarded as explanation for the persistence of odorous molecules after food consumption, and the development and/or duration of the so-called 'after-odor'. The opposite effect with odorants being no longer present in the oral cavity but being still perceived due to cognitive or receptor phenomena, cannot be excluded but has to our knowledge, not yet been shown. Generally, retronasal aroma perception of odorants released within the oral cavity is only possible when the velum-tongue barrier is opened [8]. This can occur, for example, during talking, breathing through the mouth, swallowing of saliva or often just unconsciously at rest [9]. To varying extent these actions can result in a transfer of aroma-loaded air and/or saliva into the pharynx, depending on the type and extent of action performed. From the pharyngeal areas, the air is further transported by the tidal breath-flow into the nasal cavity and to the olfactory epithelium. The odorants from liquid foods have access to the nasal cavity mainly after the swallowing action producing an aroma-rich 'Swallow-breath'.

Land [10] and Buettner et al. [8] reported this 'Swallow-breath' as the main source of aroma compounds in the nasal cavity originating via the retronasal route. However, additional aroma peaks could be demonstrated by further swallowing actions of saliva and traces of the liquid beverage, as well as distinct tasting actions [4,5]. For some food textures, it was also demonstrated that food material can form a food matrix lining on the throat, and can further deliver odorants via the retronasal route [7]. In agreement with this, a recent modeling study by Normand et al [11] suggests that extended retronasal aroma perception of liquid samples originates from the throat lining. In Normand's models, the kinetics of the release of aroma compounds during drinking is divided into three parts. First, the swallowing breath results in a small amount of aroma-rich air being transferred to the nose. Secondly, in the next few breaths, the release originates from the liquid film coating the throat. In the third

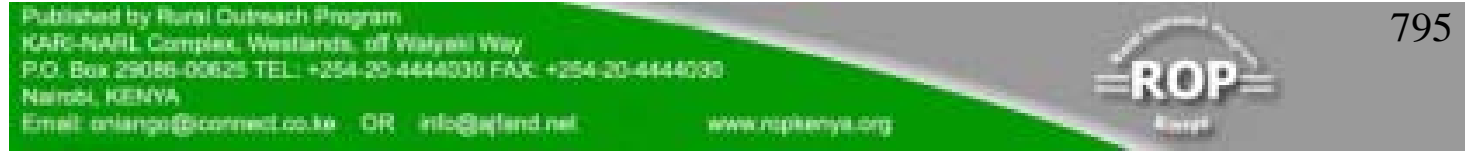


phase, the interaction with the mucosa must be considered. This is in agreement with data presented by Buettner [5] on oral and pharyngeal aroma perception after wine consumption. In a further consequence, aroma compounds diffuse from the saliva to the air and mucosa. Then, after the concentration in the mucosa reaches equilibrium with decreasing concentration in saliva, compounds are released from the mucosa to the saliva and air phases. Therefore, the importance of the aroma portion that is adsorbed by the mucosa and its effect on retronasal aroma is of interest [12]. The aims of the present investigation were first to identify and quantify odorants exhaled during palm wine consumption and secondly, to determine the influence of odorant adsorption or desorption on aroma persistence.

\section{MATERIALS AND METHODS}

\section{Materials}

Three bottles of palm wine (E. guineesis) $(1.5 \mathrm{~L})$ were freshly purchased directly from the production farm in a sterilized container encrushed in ice. The samples were bottled and pasteurized, dispensed into $45 \mathrm{~mL}$ glass-tubes, and stored at $-18^{\circ} \mathrm{C}$ prior to analysis. Accordingly, there was no extended storage at elevated temperatures of samples prior to analysis.

\section{Chemicals}

The following odorants were obtained from the suppliers shown: methyl butanoate, 99\%; 2/3-methyl 1-butanol, 98\%; ethyl hexanoate, 79\%; acetoine, 98\%; ethyl lactate, 99\%; 3-isobutyl 2-methoxypyrazine, 70\%; 3-methylbutyl acetate, 99\%; linalool, 98\%; butanoic acid 97\%; 3-methylthio 1-propanal (methional), 98\%; 4-hydroxy-2,5dimethyl-3(2H)-furanone, 98\%; 3-methoxyphenol, 98\%; ethyl cinnamate, 98\%; phenylacetic acid, 99\%; 2/3-methylbutanal, 98\%; ethyl 2-methylbutanoate, 99\%; (E,E)-nona-2,4-dienal, 99\%; (E,E)-deca-2,4-dienal, 99\%; hexan-1-ol 98\%; $\gamma$ dodecalactone, 98\%; 3-hydroxy-4,5-dimethyl-2(5H)-furanone (sotolone), $98 \%$ hexyl 3-methylbutanonate, 97\% (Aldrich, Steinheim, Germany), butan-2,3-dione, 99\%; 2-acetyl pyridine, 99+\% (Fluka, Neu-Ulm, Germany); 2-phenylethanol, 99\% (Acros Organics, New Jersey, USA), acetic acid, 99\% (Merck, Darmstadt, Germany), $\beta$-damascenone, 98\% (Symrise, Holzminden, Germany); 2-ethyl-4-hydroxy-5-methyl3(2H)-furanone, $>85 \%$ (International Laboratory Limited, San Bruno, USA).

Syntheses The following compounds were synthesized according to the literature cited in brackets: (Z)-octa-1,5-dien-3-one [13], 2-acetyl-1-pyrroline [14], (Z)-non-2enal [15], (Z)-dec-2-enal [16].

The compounds were freshly distilled prior to analysis. Chemical and sensory purity was checked by high resolution gas chromatography-olfactometry (HRGC/O) as well as high resolution gas chromatography-mass spectrometry (HRGC-MS).

\section{Panelists}

Panelists (two males and two females) were non-pregnant volunteers (non-smokers) of the Technical University of Munich exhibiting no known illnesses at the time of examination, and with normal olfactory and gustatory function. Subjective aroma perception was normal in the past and at the time of examination. The panelists had a

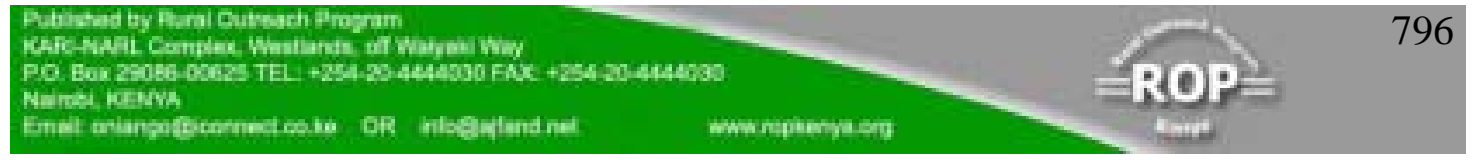


normal salivary flow and were selected for their excellent oral hygiene, thereby not suffering from oral diseases and nuisances, such as plaque, caries, tartar, gingivitis and periodontosis. In regular weekly training sessions, panelists were tested for their olfactory function with selected supra-threshold aroma solutions prior to participation in the experiment. Subjective aroma perception was normal in the past and at the time of examination, being tested with a defined set of aroma substances and an internally developed 'flavor language' [17].

Experiments were performed $2 \mathrm{~h}$ after breakfast and thorough cleaning ( $5 \mathrm{~min}$ ) of the teeth and oral cavity with an aroma-free toothpaste. Before analysis, each panelist rinsed his mouth several times with tap water to avoid any contamination, then waited for an additional $15 \mathrm{~min}$ to start.

\section{Sensory evaluation}

Assessors (five male, five female) were from the Technical University of Munich. In preceding weekly training sessions, the panelists were trained in recognizing orthonasally and retronasally 150 selected odorants at different odorant concentrations according to their odor qualities. Training in these sessions was at least for one year prior to participation in the actual sensory experiments. Sensory analyses were performed in a sensory panel room at $21 \pm 1^{\circ} \mathrm{C}$ at three different sessions. On the basis of reference aroma solutions at defined concentrations, a flavor language was developed, defining the specific smell of a compound for a certain aroma attribute. On the basis of these aroma attributes, palm wine was evaluated by the whole panel. Samples $(100 \mathrm{~mL})$ were opened and immediately applied to sensory evaluation. Palm wine $(25 \mathrm{~mL}$ each), were singly presented to the sensory panel for retronasal evaluation in covered glass vessels (capacity $45 \mathrm{~mL}$ ). The total amount of the sample was taken into the oral cavity, kept for $10 \mathrm{~s}$ with closed lips and closed velum and rinsed carefully within the oral cavity, then swallowed. At defined time intervals (2fold increase) after swallowing (10,20,40, and $80 \mathrm{~s})$ the intensities of the overall retronasal aroma perception as well as those of the single predefined odor qualities were rated on a nine-point scale from 0 (not perceivable), 1 (detection level), 2 (recognition level), 3 (intense perception) to 4 (very intense perception) by the panelists. Rating was performed by deliberately opening the velum-tongue border exactly at these times according to the protocol described elsewhere [4]. The results obtained in three different sessions were averaged and plotted as histograms (fig. 2). The values obtained in different sessions and for the different assessors differed by not more than $10 \%$.

\section{EXOM Analysis}

Prior to oral application of the sample, the oral cavities of the panelists were screened for odorants ("blank"). In all subsequent analyses, values obtained were corrected for trace odor contaminants.

Odorants in breath exhaled from the nose during the consumption of palm wine (25 $\mathrm{mL} / \mathrm{swallow}$, total volume $250 \mathrm{~mL}, 5 \mathrm{~min}$ consumption, $1 \mathrm{swallow} / 0.5 \mathrm{~min}$ ) and from blank breath were analyzed by a modified EXOM analysis [12]. The air being exhaled immediately after swallowing of the palm wine, the so-called 'swallow breath', as well

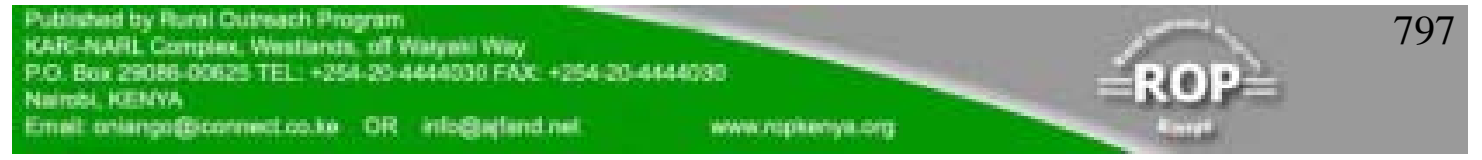




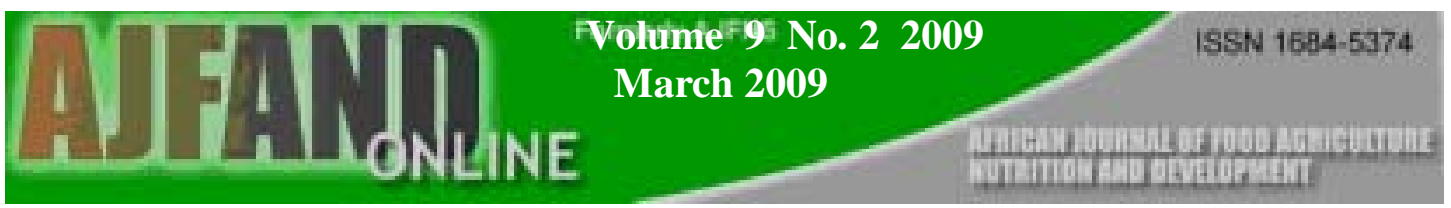

as that exhaled $20 \mathrm{~s}$ after swallowing, was cryo-focused with liquid nitrogen in a specially designed glass apparatus (fig 1), thereby avoiding the trapping of laboratory air. The cryo-focused exhaled air was eluted with dichloromethane $(100 \mathrm{~mL})$. Quantification was performed by spiking with known amounts of the respective stable isotope labeled standards and after stirring for equilibration $(20 \mathrm{~min})$. The extracts were concentrated to a final volume of $200 \mu \mathrm{L}$ by means of concentration on a Vigreux-coloumn and subsequent microdistillation [18], and were subsequently analyzed by two-dimensional HRGC-MS and HRGC-Olfactometry (HRGC-O).

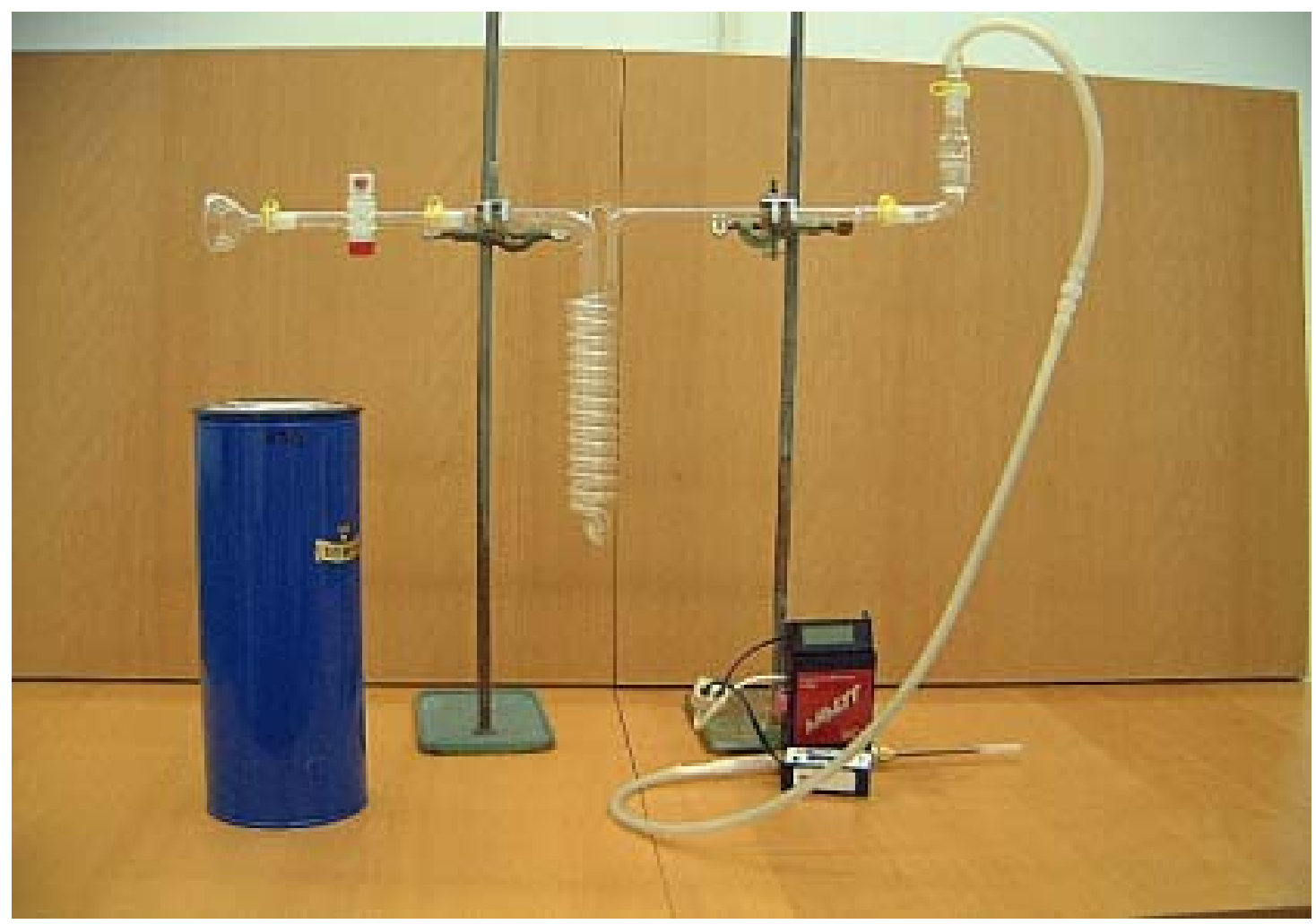

Figure 1: Breath cryofocussing device.

\section{High resolution gas chromatography - mass spectrometry}

The odorants were quantified by two dimensional gas chromatography (TD-HRGC) using a mega 2 gas chromatograph (Fisons Instruments, Mainz-Kastel, Germany) as the pre-column system in tandem with a Fisons GC 5160 as the main column system. MS analyses were performed with an ITD-800 (Fisons Instruments, Mainz-Kastel, Germany) running in the CI-mode with methanol as the reagent gas. The following fused silica columns were used: DB-FFAP (30 m x $0.32 \mathrm{~mm}$ i.d., film thickness 0.25 $\mu \mathrm{m}, \mathrm{J} \& \mathrm{~W}$ Scientific, Folsom, USA) and DB-5 (SE-54; $30 \mathrm{~m} \times 0.32 \mathrm{~mm}$ i.d., film thickness $0.25 \mu \mathrm{m}, \mathrm{J} \& \mathrm{~W}$ Scientific). The gas chromatographic conditions were the same as described previously [18]. 


\section{Aroma dilution analysis (ADA)}

Freshly prepared palm wine samples $(15 \mathrm{~mL})$ were equilibrated in a septum-sealed vessel (100 $\mathrm{mL}$ total volume) for $30 \mathrm{~min}$ at room temperature. Preliminary sensory experiments had shown that no significant overall odor change was observable within this storage time. Using fresh portions of wine in each experiment, decreasing headspace volumes $(10-0.32 \mathrm{~mL}$, decrease factor two) were taken off by means of gas-tight syringes, then cryofocussed on a fused silica trap (TCT-PTI-system 4001; Chrompack, Mühlheim, Germany) and finally injected onto a fused silica column DB5 (SE-54; $30 \mathrm{~m} \times 0.32 \mathrm{~mm}$, film thickness $0.25 \mu \mathrm{m} ; \mathrm{J} \& \mathrm{~W}$ Scientific) [19]. After injection, the temperature of the oven was held at $0{ }^{\circ} \mathrm{C}$ for $2 \mathrm{~min}$, then raised at 6 ${ }^{\circ} \mathrm{C} / \mathrm{min}$ to $200{ }^{\circ} \mathrm{C}$. At the end of the column, the effluent was split 1:1 (by vol.) onto two uncoated but deactivated fused silica capillaries $(50 \mathrm{~cm} \times 0.32 \mathrm{~mm})$ leading to an FID and a sniffing port. The perceived odors were attributed to the odorants identified in the preceding experiments using the solvent extract, by means of odor quality, odor intensity, and retention index [3]. Identification was further based on mass spectrometric identification as described in the following section.

The Flavor Dilution Factors (FD) of the odor-active compounds given in Table 1 were calculated by dividing the largest volume analysed $(10 \mathrm{~mL} ; \mathrm{FD}=1$ by definition) by the lowest volume in which the respective odorant was yet detectable. In total, three experienced sniffers were used to perform the ADA experiments. Their responses (sensitivity) to the individual compounds did not differ by $>2$ FD factors.

\section{Identification of Volatile Compounds}

Compounds were identified by comparison with the reference substances on the basis of the following criteria: retention index (RI) on two stationary phases of different polarities, mass spectra obtained by MS (EI) and MS (CI), and odor quality as well as odor intensity perceived at the sniffing port. Odor intensity was checked by GC/O and by comparing the FID signal caused by a defined amount of each reference aroma compound. 


\section{RESULTS}

\section{Static headspace analysis/olfactometry (SHO)}

Odorants present in the headspace above a food are assumed to render the so - called "top - note" to a food [20]. By application of the static headspace/olfactometry (SHO) - technique, most specifically the Aroma Dilution Analysis [21], odorants contributing to this "top - note" can be evaluated. The results of an application of the ADA experiments on palm wine are summarized in Table 1. 19 odorants were identified in the headspace above the palm wine. These include for example six esters, four alcohols, three carbonylic compounds, two acids, two heterocyclic compounds and one aromatic compound. Among the compounds detected, the buttery smelling acetoine, the popcorn-like smelling 2-acetyl-1-pyrroline, and the earthy-bell pepperlike smelling 3-isobutyl-2-methoxypyrazine were the most potent odorants in Headspace-HRGC-O with FD factors of 256 and 128, respectively. Additional compounds with high FD factors were the banana-like smelling 3- methylbutyl acetate as well as 2-phenylethanol with honey-like aroma impression. Other relatively odoractive compounds were fruity, sweet and flowery compounds such as ethyl hexanoate, linalool, phenylacetic acid, and ethyl 2-methylbutanoate, while malty, buttery or cooked-potato-like impressions originated e.g. from the 2- and 3-methylbutanols, butan-2,3-dione and 3-methylthio propanal.

\section{Sensory evaluation}

In the following, the retronasal aroma impressions from palm wine consumption should be evaluated both by means of sensory, as well as analytical analyses. Freshly opened palm wine was evaluated retronasally as described in the experimental section. Palm wine exhibits a very specific and gradually changing retronasal aroma profile [3] that was described by the sensory panel as detailed below. Palm wine aliquots $25 \mathrm{~mL}$ were taken into the oral cavity, kept for $10 \mathrm{~s}$ with closed lips and rinsed carefully within the oral cavity, then swallowed. First, the wine was evaluated with regard to the initial retronasally perceived intensities of predefined aroma attributes when introduced into the mouth. Then, their retronasal sensory persistence was profiled as described in the sensory evaluation at defined time intervals. The following odor qualities, fruity, citrusy, yeast - like, acidic, nutty and popcorn-like were selected as descriptors based on preliminary sensory evaluation (data not shown). Fruity and citrusy were the most intense aroma qualities perceived upon sample introduction into the mouth (fig. 2). Swallowing of the palm wine elicited a more acidic impression followed by citrusy, fruity nuances respectively. At 10 and 20 sec after swallowing, panelists described an increase in the nutty and popcorn-like aroma impressions. From the changes in the aroma profiles with time, it is evident that at last the popcorn-like and nutty notes persisted longer than the other notes. 
(a)

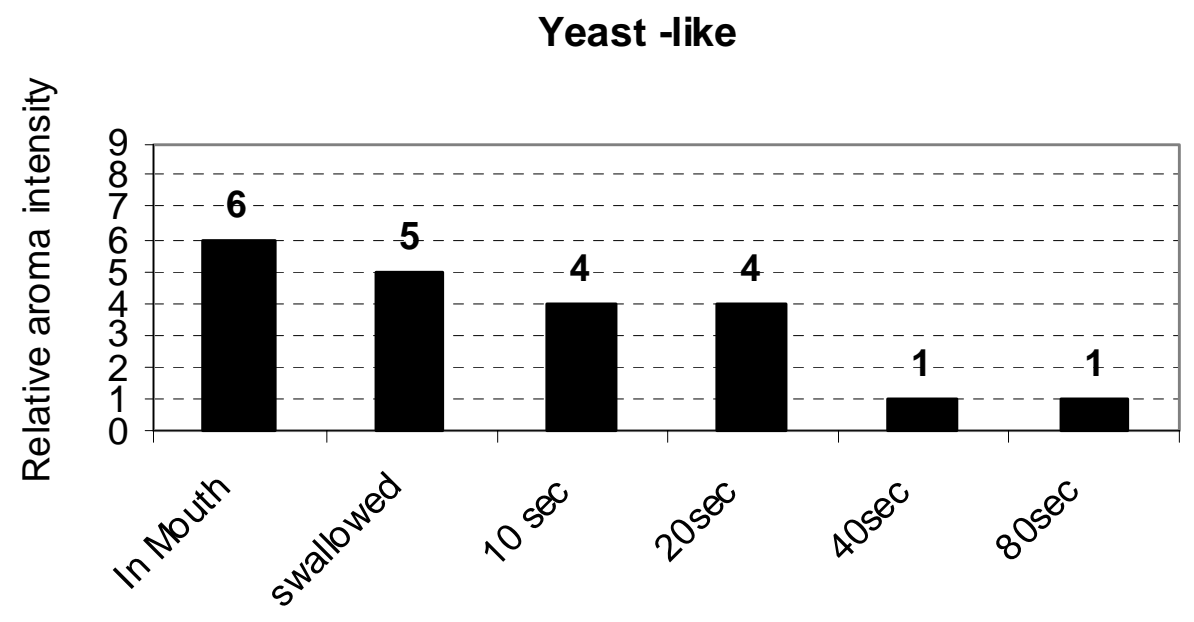

b)

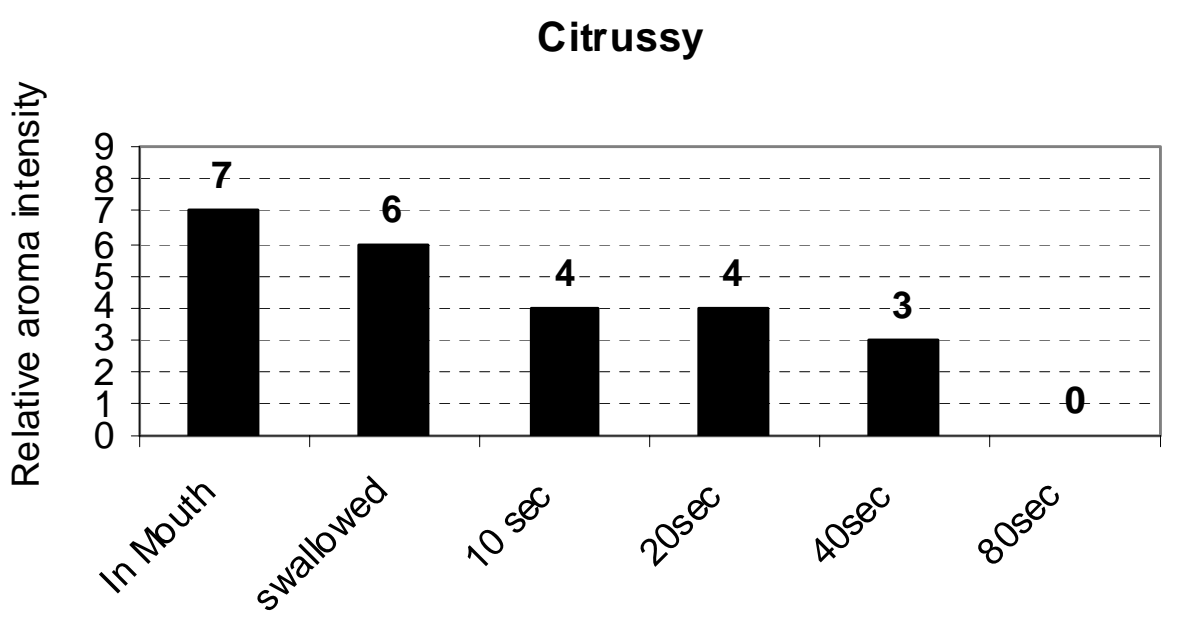

Figure 2: Time-resolved retronasal aroma evaluation from swallowing of Palm wine The aroma intensity is rated on a nine-point scale from 0 (not perceivable) to 4 (very intense) with half-point steps. 
(c)

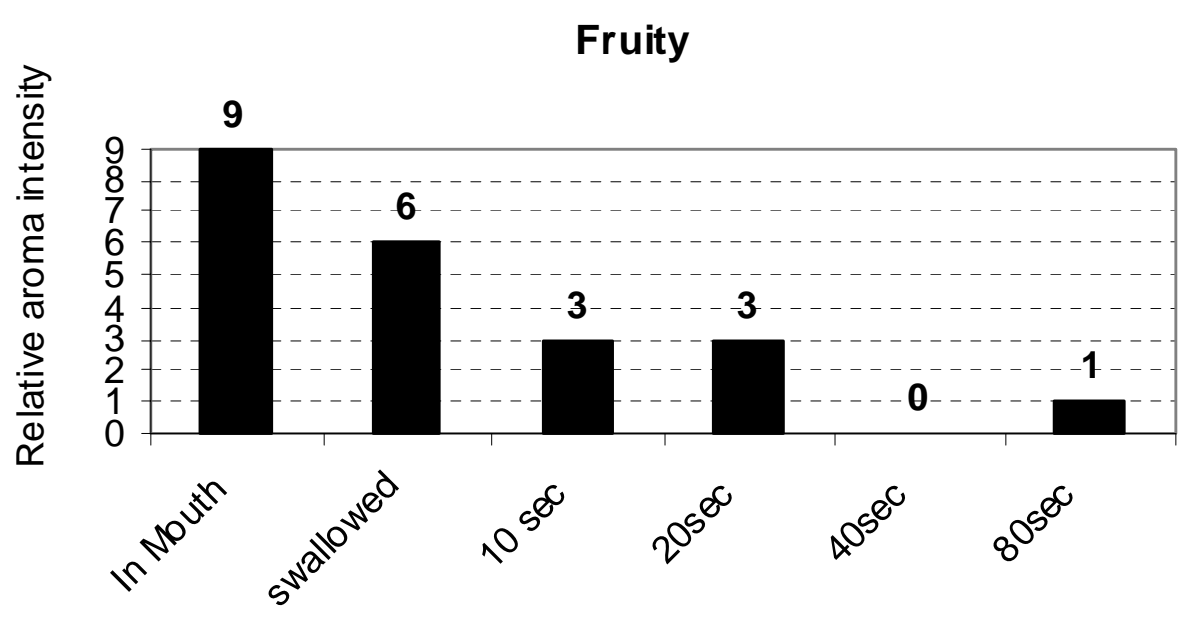

(d)

Acidic

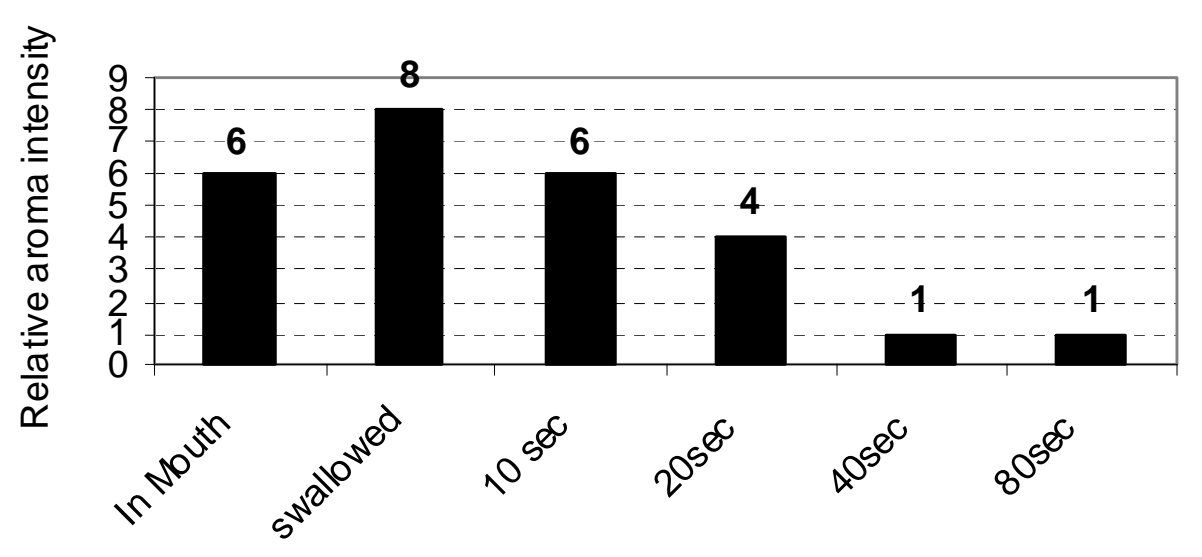

Figure 2: continued. 
(e)

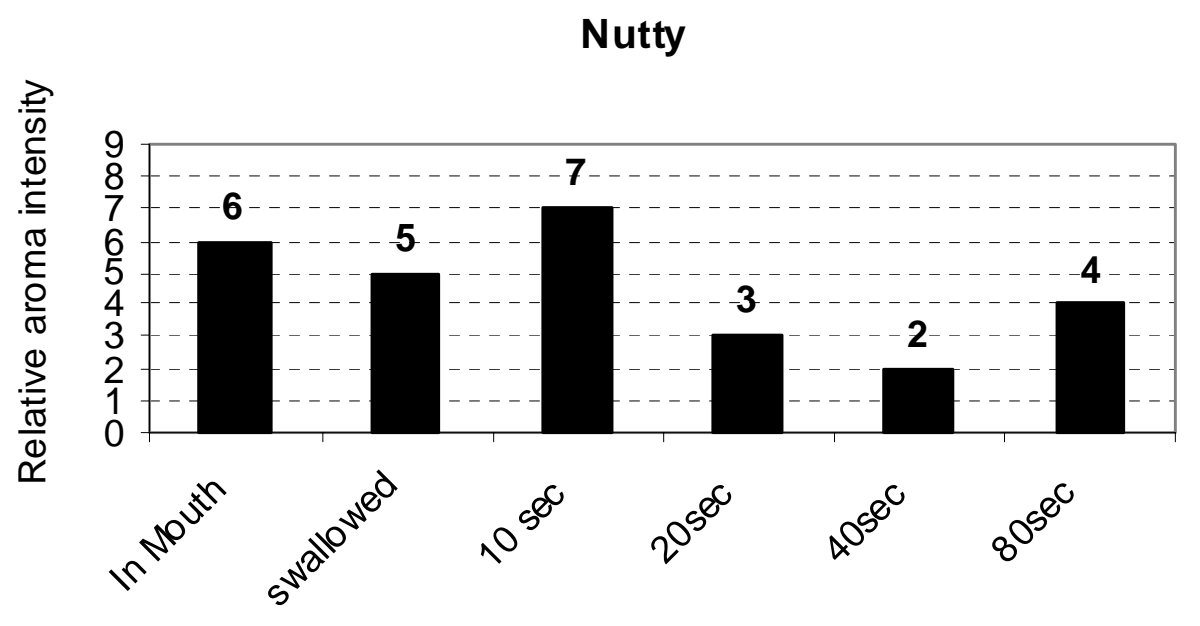

(f)

\section{Popcorn}

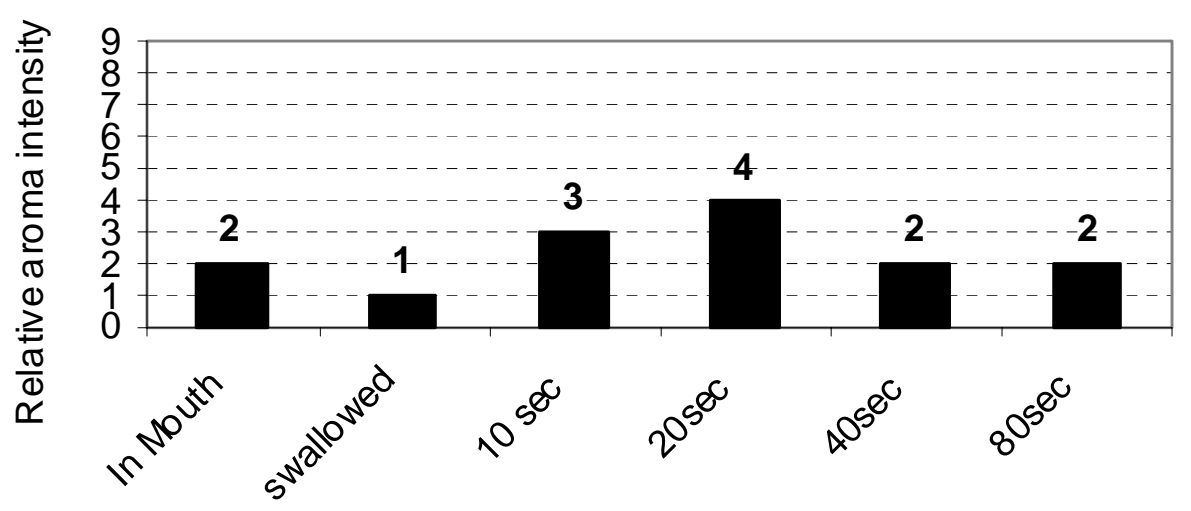

Figure 2: continued. 


\section{Determination of exhaled odorants during palm wine consumption by modified EXOM analysis}

In the following, the results from the retronasal sensory evaluation should be compared to analytical data obtained from EXOM analysis.

As basis for EXOM analysis, blank breath was first evaluated with regard to specific odor compounds. When screening control breath (blank breath) of the participants by smelling each others breaths prior to palm wine consumption, all panelists reported a faint buccal smell which was described as a bit tallowy, slightly acidic and as the typical oral smell of healthy people. It was described as only perceivable when directly sniffing the panelists mouths and was not attributed to any increased oral smell as induced by, for example, halitosis. Screening of the untreated oral cavities of the participants by means of EXOM - approach revealed a weak detection of ten odor-active compounds which were detectable at each sampling day (Table 2, and compounds indicated with BB (blank breath) in Table 3). Six of these compounds, oct-1-en-3-one, acetic acid, $(Z)$-non-2-enal, $(Z)$-dec-2-enal, $(E, E)$-deca-2,4-dienal, and $\gamma$-dodecalactone were identified, and their presence in the oral cavity could be verified.

Table 3 shows the results of odor qualities and retention indices of exhaled odorants during different stages of palm wine consumption as detected by means of the modified EXOM analysis coupled with HRGC-O. Aroma compounds in blank breath and in breath exhaled from the nose after swallowing of palm wine $(25 \mathrm{~mL} / \mathrm{swallow}$, total volume $250 \mathrm{~mL}, 5 \mathrm{~min}$ consumption, 1 swallow/ $0.5 \mathrm{~min}$ ) were first analyzed [12]. The exhaled air was cryofocused with liquid nitrogen in a specially designed glass apparatus (fig. 1) thereby avoiding the trapping of laboratory air. Subsequently, the samples were extracted with dichloromethane at room temperature as described in the experimental section, concentrated and analyzed by two-dimensional high resolution gas chromatography-olfactometry and mass spectrometry.

This approach led to the detection of most of the odorants that were previously found either by static headspace analysis-olfactometry (Table 1) or by means of aroma extract dilution analysis [3].

Generally, a total of 24 odorants were detected in the so - called "swallow breath" (immediately after consumption), while only 14 odorants were detected after $20 \mathrm{~s}$ of swallowing. The detected odorants belonged to diverse substance classes such as esters, carbonyl compounds such as aldehydes and ketones, alcohols and heteroaromatic compounds, and others. Potent esters were e.g. methyl butanoate, ethyl butanoate, ethyl pentanoate, ethyl 2-methyl butanoate, ethyl hexanoate, hexyl 3methyl butanoate, 3-methylbutyl acetate, and ethyl cinnamate. Carbonyl, alcohol or aromatic compounds were e.g. 2,3-butandione, 2/3-methylbutanol, acetoine, 2acetyl 1-pyrroline, linalool, and 3-isobutyl 2-methoxypyrazine.

When looking at the total durations of detection of the odorants persisting in the breath, some significant differences become evident. First of all, the buttery smelling 2,3-butandione, the fruity smelling ethyl pentanoate, the malty 2/3-methyl butanol, the 
buttery smelling acetoine, 2-acetyl 1-pyrroline, $\gamma$-dodecalactone, linalool, and the earthy smelling 3-isobutyl 2-methoxypyrazine were detected for longer time intervals after intra-oral application of the palm wine. In contrast to this, the persistence of the following odorants, methyl butanoate, ethyl butanoate, ethyl 2-methylbutanoate, ethyl hexanoate, methional, hexyl 3-methylbutanoate, 3-methylbutyl acetate, $\beta$ damascenone, 2-methoxyphenol, 2-ethyl-4-hydroxy-5-methyl-3(2H)-furanone and ethyl cinnamate was reduced. This probably accounted for the absence of these odorants from the exhaled breath air, $20 \mathrm{~s}$ after swallowing of palm wine.

Interestingly, 4 odorants which were not earlier detected in the "swallow breath" were found in the exhaled air $20 \mathrm{~s}$ after swallowing. These include 2-acetyl 1-pyrroline, with a characteristic popcorn note, linalool and two unknown odor-active compounds with characteristic citrusy and buttery notes, respectively. Especially 2-acetyl 1pyrroline was detected as very intense compound at $20 \mathrm{sec}$ after swallowing in exhaled breath.

\section{DISCUSSION}

The results of the static headspace analysis indicate that the detected 19 compounds, which had also been detected as key odorants in solvent extracted palm wine [3], are important contributors to the specific 'top-note' of palm wine. Earlier reports have shown that odorants rendering the top note in the headspace above a food are most probably identical with those perceived orthonasally via the nostrils [12]. As most potent among these for Palm wine orthonasal aroma impression, the popcorn-like smelling 2-acetyl 1-pyrroline, the earthy-bell pepper-like smelling 3-isobutyl 2methoxypyrazine and the banana-like smelling 3-methylbutyl acetate as well as 2phenylethanol with honey-like aroma impression have to be specifically mentioned.

Regarding the results from EXOM-HRGC-O analysis, it has to be noted that especially the polar or acidic compounds, as well as some compounds with relatively high molecular weights were not detected. Still, they were previously identified by means of AEDA. This might indicate that these substances play only a minor or no role in retronasal aroma perception of palm wine.

Examples are butanoic acid and phenyl acetic acid (previously identified in palm wine samples by means of ADA as well as AEDA), acetic acid, methyl propanoic acid, pentanoic acid, and 3-methylpentanoic acid (previously identified by AEDA only). Other compounds that were not detectable in the exhaled breath by EXOM-HRGC-O but by ADA or AEDA, respectively, were ethyl lactate, diethyl succinate, 1-hexanol, 3-methylthio 1-propanol, 2-phenylethanol, vanillin, as well as the furanone compounds 4-hydroxy-2,5-dimethyl-3(2H)-furanone, 3-hydroxy-4,5-dimethyl-2(5H)furanone, 2-ethyl 3,5-dimethylpyrazine, 2-acetylpyridine, and 4-methoxy-2methylphenol. Probable reasons are that these substances are quite polar, but also that the buffering capacity of the saliva is relatively high. Therefore, perception of these compounds is expected to be reduced. Another reason can be relatively low odor potencies of compounds in the original palm wine samples as for $(Z)$-octa-1,5.dien-3one and $(E, E)$-nona-2,4-dienal which gave detection with very low FD factors in AEDA.

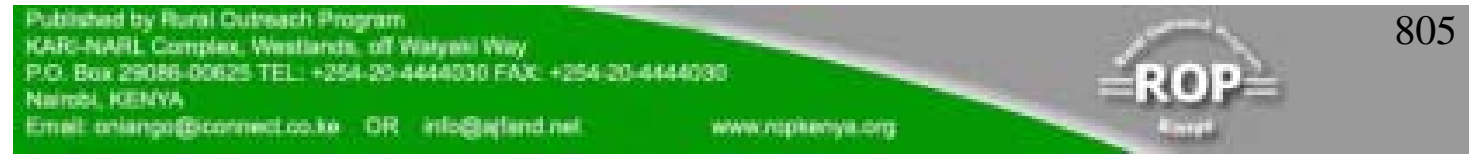


The majority of compounds which were absent in the exhaled breath after $20 \mathrm{~s}$ of swallowing were esters and aldehydes. One possible factor responsible for this might be the degradation of esters by hydrolysis and aldehyde-reduction, as many esterolytic enzymes can be found in human saliva [22], and as odorant metabolism in the presence of saliva has been reported previously [5]. However, the time interval relevant in this study (up to $20 \mathrm{sec}$ after swallowing) is relatively short so that it is not clear to which extent metabolic activity accounts for the observed effect. On the other hand, different volatility or partitioning differences between mucosal or salivary media and the air phase might be further reasons [23]. The influence of the diverse parameters can not be derived from the presented EXOM data only.

Increased detection was found for four odorants which were not earlier detected in the "swallow breath" but were found in the exhaled air $20 \mathrm{~s}$ after swallowing, 2-acetyl 1pyrroline, linalool and the two unknown odor-active compounds with characteristic citrusy and buttery notes. We assume that this might be due to release phenomena correlating with intra-oral changes in $\mathrm{pH}$ from the relatively acidic $\mathrm{pH}$ of palm wine to the $\mathrm{pH}$ of saliva. Another reason might be metabolic processes or generation from precursors. However, the underlying principles were not studied in further detail within this investigation.

Generally, both EXOM and sensory evaluation (fig. 2) mirror to a certain extent the higher or increased persistence and perception of nutty, popcorn, and yeast-like odor notes from palm wine (mainly represented by 2-acetyl 1-pyrroline, acetoin, and 2/3methylbutanol). The aroma changes perceived with time did not only result from a release of some compounds with later on - set (starting point) but also from odorants being detectable right from the starting point of the analysis of 'afterodor' [4]. That means, changes were also likely to be induced by the faster removal of some odorants from the oral cavity while others persisted for longer time. As a consequence, the perception of these persisting compounds became more dominant as the short - lasting odorants were removed at later times. Delahunty et al [24] earlier reported that potential flavor compounds in foods have different physical and chemical properties, and only those compounds which achieve a sufficient concentration in the vapour phase or aqueous phase to stimulate the olfactory and lingual receptors can have a direct impact on flavor. The concentration of a flavor compound reaching these receptors is influenced by the rate of its release from the food and adsorption to the oral mucosa. 


\section{CONCLUSION}

The static headspace/olfactometry (SHO) of palm wine revealed 19 odor - active compounds most likely constituting the 'top-note' of palm wine. However, the 'swallow breath' (immediately after swallowing) produced an initial 24 odor-active compounds; of all these compounds, only 14 persisted in the exhaled breath $20 \mathrm{~s}$ after swallowing. Dynamic changes were observed both in retronasal sensory evaluation as well as in EXOM analysis.

\section{Acknowledgement}

We gratefully acknowledge the support of this work by Prof. Schieberle, by the Deutsche Forschungsanstalt fuer Lebensmittelchemie, and the Alexander von Humboldt Foundation for the fellowship awarded to Dr. Lasekan. The work of Dr. Buettner was supported by the Hochschulwissenschaftsprogramm HWP II, Technical University of Munich.

\section{ABBREVIATIONS}

ADA

AEDA

BB

FD

HRGC-MS
Aroma Dilution Analysis

Aroma Extract Dilution Analysis

Blank Breath

Flavor Dilution

High resolution gas chromatography mass spectrometry 
Table 1: Static headspace analysis/olfactometry (SHO/O) of palm wine, and aroma dilution analysis (ADA).

\begin{tabular}{|c|c|c|c|c|}
\hline No. & Odorants $^{\text {a }}$ & Odor quality ${ }^{\text {b }}$ & $\begin{array}{l}\text { Retention index } \\
\text { on SE-54 }\end{array}$ & $\begin{array}{l}\text { FD } \\
\text { factor }\end{array}$ \\
\hline 1. & Butan-2,3-dione & Buttery & 592 & 32 \\
\hline 2. & 2/3-Methyl butanol & Malty & 737 & 64 \\
\hline 3. & Butanoic acid & Sweaty-buttery & 821 & 8 \\
\hline 4. & Acetoine & Buttery & 852 & 256 \\
\hline 5. & Ethyl 2-methyl butanoate & Fruity & 852 & 32 \\
\hline 6. & 3-Methylbutyl acetate & Banana-like & 878 & 128 \\
\hline 7. & Ethyl pentanoate & Sweet-fruity & 900 & 16 \\
\hline 8. & 3-Methylthio propanal & Cooked potato-like & 905 & 32 \\
\hline 9. & 2-Acetyl 1-pyrroline & Popcorn-like & 922 & 256 \\
\hline 10. & Methyl butanoate & Sweet-fruity & 982 & 16 \\
\hline 11. & Ethyl hexanoate & Fruity & 1001 & 64 \\
\hline 12. & Linalool $^{\mathrm{c}}$ & Fresh-flowery & 1102 & 64 \\
\hline 13. & $\begin{array}{l}\text { 3-Hydroxy-4,5-dimethyl- } \\
2(5 H) \text {-furanone }\end{array}$ & Spicy, savory-like & 1109 & 8 \\
\hline 14. & 2-Phenylethanol & Honey-like & 1117 & 128 \\
\hline 15. & 3-lsobutyl 2-methoxypyrazine & $\begin{array}{l}\text { Earthy, } \\
\text { bell pepper-like }\end{array}$ & 1175 & 256 \\
\hline 16. & Hexyl 3-methylbutanoate & Fruity & 1244 & 16 \\
\hline 17. & Phenylacetic acid & Honey & 1262 & 64 \\
\hline 18. & $\beta$-Damascenone & Flowery & 1389 & 16 \\
\hline 19. & Vanillin $^{\mathrm{c}}$ & Vanilla & 1404 & 16 \\
\hline
\end{tabular}

\footnotetext{
${ }^{\mathrm{a}}$ The compounds were identified by comparing their mass spectra, retention index, and odor quality with reference compounds.

${ }^{\mathrm{b}}$ Odor quality as perceived at the sniffing port.

${ }^{c}$ MS signals were too weak for an unequivocal interpretation. The compounds were identified on the basis of the remaining criteria in footnote (a).
} 
Table 2: $\quad$ Exhaled odorant measurement (EXOM) of the oral cavity of a healthy panelist prior to palm wine consumption (blank).

\begin{tabular}{lllll}
\hline & & & \multicolumn{2}{l}{ Retention index } \\
No. & Odorant $^{\text {a }}$ & Odor quality $^{\mathbf{b}}$ & FFAP & SE-54 \\
\hline 1. & Oct-1-en-3-one & Mushroom-like & 1297 & 981 \\
2. & Acetic acid & Acidic, vinegar-like & 1450 & $\mathrm{nd}^{\mathrm{c}}$ \\
3. & Unknown & Phenolic & 1484 & $\mathrm{nd}^{\mathrm{c}}$ \\
4. & $(Z)$-Non-2-enal & Fatty, tallowy & 1503 & 1148 \\
5. & $(Z)$-Dec-2-enal & Fatty, tallowy & 1591 & 1250 \\
6. & $(E, E)-$ Deca-2,4-dienal & fatty & 1684 & 1215 \\
7. & Unknown & Malty & 1748 & $\mathrm{nd}^{\mathrm{c}}$ \\
8. & Unknown & Soapy & 1997 & $\mathrm{nd}^{\mathrm{c}}$ \\
9. & Unknown & Sweet & 2150 & $\mathrm{nd}^{\mathrm{c}}$ \\
10. & $\gamma$-Dodecalactone & Coconut-like & 2376 & 1684 \\
\hline
\end{tabular}

a The compounds were identified by comparison with the respective reference substances on the basis of the following criteria: retention indices on different stationary phases given in the table, mass spectra obtained by MS (EI) and MS (CI), and odor quality as well as odor intensity perceived at the sniffing port.

${ }^{\mathrm{b}}$ Odor quality perceived at the sniffing port.

${ }^{\mathrm{c}}$ nd: Not determined. 


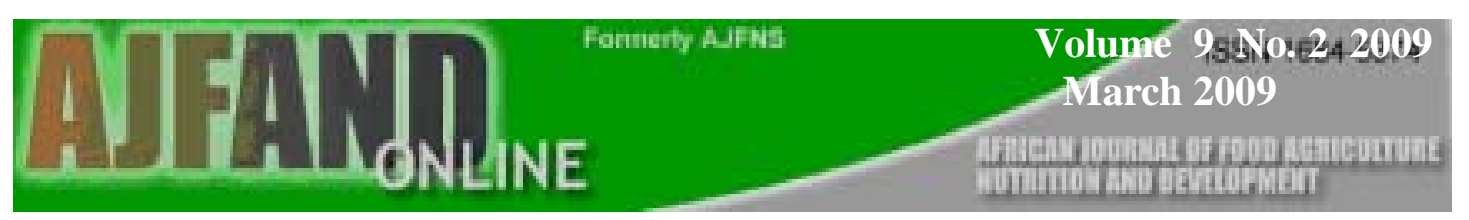

Table 3: $\quad$ Exhaled odorant measurement (EXOM) during palm wine consumption.

\begin{tabular}{|c|c|c|c|c|c|c|c|}
\hline \multirow[b]{2}{*}{ No. } & \multirow[b]{2}{*}{ Odorant $^{a}$} & \multirow[b]{2}{*}{$\begin{array}{l}\text { Previously } \\
\text { identified by }\end{array}$} & \multirow[b]{2}{*}{ Odor quality ${ }^{c}$} & \multicolumn{2}{|c|}{$\underline{\text { Retention index }}$} & \multicolumn{2}{|c|}{$\begin{array}{l}\text { Exhaled breath } \\
\text { odorants at }\end{array}$} \\
\hline & & & & FFAP & SE-54 & $0 \sec ^{d}$ & $20 \sec ^{\mathrm{e}}$ \\
\hline 1. & Methyl butanoate & ADA, AEDA & Sweet-fruity & 981 & 723 & + & - \\
\hline 2. & Butan-2,3-dione & ADA, AEDA & Buttery & 993 & 592 & + & + \\
\hline 3. & Ethyl butanoate & & Fruity & 1028 & 803 & + & - \\
\hline 4. & Ethyl 2-methylbutanoate & ADA, AEDA & Fruity & 1040 & 852 & + & - \\
\hline 5. & Ethyl pentanoate & ADA, AEDA & Sweet-fruity & 1067 & 900 & + & + \\
\hline 6. & 2/3-Methylbutanol & ADA, AEDA & Malty & 1215 & 737 & + & + \\
\hline 7. & Ethyl hexanoate & ADA, AEDA & Fruity & 1226 & 1001 & + & - \\
\hline 8. & Acetoine & ADA, AEDA & Buttery & 1275 & 852 & + & + \\
\hline 9. & Oct-1-en-3-one & $\mathrm{BB}$ & Mushroom-like & 1297 & 981 & + & + \\
\hline 10. & 2-Acetyl 1-pyrroline & ADA, AEDA & Popcorn & 1323 & 922 & - & + \\
\hline 11. & Unknown & Not detected & Citrusy & 1337 & nd & - & + \\
\hline 12. & Unknown & Not detected & Fatty & 1376 & nd & + & - \\
\hline 13. & Unknown & Not detected & Buttery & 1397 & nd & - & + \\
\hline 14. & Hexyl 3-methylbutanoate & ADA, AEDA & Fruity & 1430 & 1244 & + & - \\
\hline 15. & 3-Methylthio propanal & ADA, AEDA & Cooked potato & 1460 & 905 & + & - \\
\hline 16. & (Z)-Non-2-enal & $\mathrm{BB}$ & Fatty, tallowy & 1503 & 1148 & + & + \\
\hline 17. & $\begin{array}{l}\text { 3-Isobutyl-2-methoxy- } \\
\text { pyrazine }\end{array}$ & ADA, AEDA & Earthy & 1517 & 1175 & + & + \\
\hline
\end{tabular}




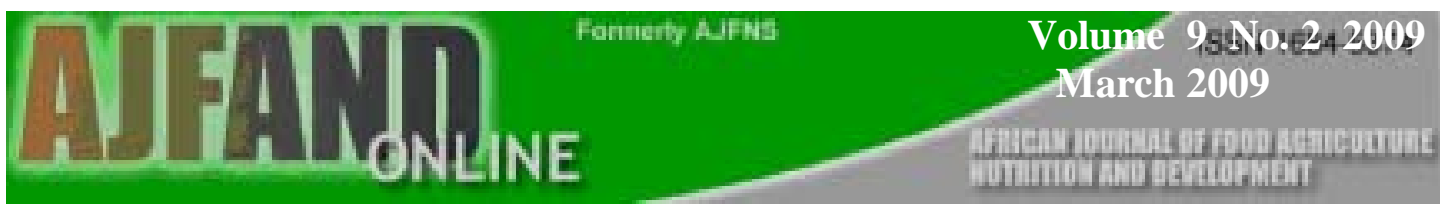

18. 3-Methylbutyl acetate

ADA, AEDA

Banana

19. Linalool

ADA, AEDA

Fresh-flowery

20. (Z)-Dec-2-enal

$\mathrm{BB}$

Solvent

AEDA

21. 2/3-Methylbutanoic acid

ADA, AEDA

22. $\beta$-Damescenone

AEDA

23. 2-Methoxyphenol

AEDA

Sweaty

Sweet-fruit

Smoky

Caramel-like

\section{7}

1540

878

1102

$1591 \quad 1250$

$1656 \quad 875$

$1823 \quad 1389$

$1880 \quad 1089$

24. 2-Ethyl-4-hydroxy-5-methyl$3(2 \mathrm{H})$-furanone

25. Ethyl cinnamate

AEDA

Sweet

2095

1159

26. $\gamma$-Dodecalactone

AEDA, BB

Coconut-like

2150

2376

1469

1684

+ Odorant detected at given time; -odorants not detected at the given time.

${ }^{a}$ The compounds were identified as reported in footnote in table 3.

${ }^{\mathrm{b}}$ Compounds were detected by means of aroma dilution analysis (ADA, Table 2), aroma extract dilution analysis (AEDA; [3]) or in blank breath (BB, Table 3).

${ }^{\mathrm{c}}$ Odor quality as perceived at the sniffing port.

${ }^{\mathrm{d}}$ Detection of exhaled aroma compounds from the nose by EXOM and HRGC-O immediately after swallowing of palm wine sample.

${ }^{\mathrm{e}}$ Detection of exhaled aroma compounds from the nose by EXOM and HRGC-O 20 seconds after swallowing.

${ }^{\text {nd }}$ Not determined.

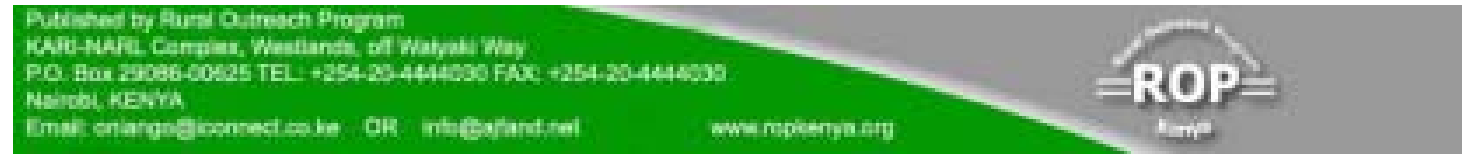




\section{REFERENCES}

1 Uzochukwu SVA, Balogh E, Tucknot OG, Levis MJ and PO Ngoddy

Volatile constituents of palm wine and palm sap. J. Sci. Food Agric. 1994; 64:

$405-411$.

2 Jirovetz L, Buchbaucer G, Fleischhacker W and MB Ngassoum Analysis of the aroma compounds of two different palm wine species ('matango' and raffia) from Cameroon using SPME - GC - FID, SPME - GC. MS and Olfactory. Ernahrung/Nutrition 2001; 25: 67 - 71.

3 Lasekan O, Buettner $\mathbf{A}$ and $\mathbf{M}$ Christlbauer Investigation of Important Odorants of Palm wine (Elaeis guineensis). Food Chem. 2007; 105: 15-23.

4 Buettner A Investigation of potent odorants and afterodor development in two chardonnay wines using the Buccal Odor Screening System (BOSS). J. Agric. Food Chem. 2004; 52: 2339-2346.

5 Buettner A Aroma release and perception during consumption of food taking into account physiological aspects. Verlag Dr. Hut, Munich, Germany, ISBN 978-3-89963-559-1; 2007.

6 Starkenmann $\mathbf{C}$ Analysis of a model reaction system containing cysteine and (E)-2-methyl-2-butenal, (E)-2-hexenal, or mesityl oxide. J. Agric. Food Chem. 2003; 51: 7146-7155.

7 Buettner A, Beer A, Hannig C, Settles M and P Schieberle Physiological and analytical studies on flavour perception dynamics as induced by the eating and swallowing process. Food Qual. Pref. 2002; 13: 497 - 504.

8 Buettner A, Beer A, Hannig C and M Settles Observation of the swallowing Process by application of videofluoroscopy and realtime magnetic resonance imaging-consequences for aroma perception. Chem. Senses 2001; 26: 1211 1219.

9 Putz T and R Pabst In Sobotta, Atlas der Anatomie des Menschen. 20th edition, cd rom version 1.5; Urban \& Fischer, Munich, 1998.

10 Land OG Perspectives on the effects of interactions on flavor perception: an overview. In: McGorrin RJ and J Leland (Eds). Flavor - Food Interaction. ACS Symposium Series 633, Washington, ACS; 1994; 2 - 11.

11 Normand V, Avison S and A Parker Modeling the kinetics of flavor release during drinking. Chem. Senses 2004; 29: 235 - 245.

12 Buettner A and P Schieberle Exhaled odorant measurement (EXOM) - a new approach to quantify the degree of in - month release of food aroma compounds. Lebensm.-Wiss. Technol. 2000; 33: 553 - 559. 
13 Ullrich F and W Grosch Identification of the most intense odour compounds formed during autoxidation of methyl linolenate at room temperature. J. Am. Oil Chem. Soc. 1988; 65: 1313-1317.

14 Buttery RG, Ling LC and BO Juliano 2-Acetyl-1-pyrroline: an important component of cooked rice. Chem. Ind. 1982; 36: 958-959.

15 Ullrich $\mathbf{F}$ and $\mathbf{W}$ Grosch Flavour deterioration of soya-bean oil: identification of intense odour compounds formed during flavour reversion. Fat Sci. Technol. 1988; 90: 332-336.

16 Kerscher $\mathbf{R}$ and W Grosch Comparative evaluation of potent odorants of boiled beef by aroma extract dilution and concentration analysis. Z. Lebensm. Unters. Forsch. 1997; 204: 3-6.

17 Czerny M, Christlbauer M, Granvogl M, Fischer A and P SchieberleP Orthonasal odor thresholds in water and odor qualities of important food aroma compounds. in preparation.

18 Buettner A and P Schieberle Evaluation of key aroma compounds in handsqueezed grapefruit juice (Citrus paradise Macfayden) by quantitation and flavor reconstitution experiments. J. Agric.Food Chem. 2001; 49: 1358-1363.

19 Schieberle P Primary odorants of pale lager beer. Differences to other beers and changes during storage. Z. Lebensm- Unters. Forsch. 1993; 193: 558 -565.

20 Hinterholzer A and P Schieberle Identification of the most odour - active volatiles in fresh hand - extracted juice of Valencia late oranges by odour dilution technique. Flav. Fragr. J. 1998; 13: $49-55$.

21 Guth H and W Grosch Quantitation of potent odorants of virgin olive oil by stable isotope dilution assays. J. Am. Oil Chem. Soc. 1993; 70: 513 - 518

22 Chauncey HH, Lionetti F, Winer RA and VF Lisanti Enzymes in human saliva. I. The determination, distribution and origin of whole saliva enzymes. $J$. dent. Res. 1954; 33: 321-334.

23 Buettner A and P Schieberle Changes in the concentrations of key fruit odorants induced by mastication. In: Roberts DD and AJ Taylor (Eds). Flavor Release. ACS Symposium Series 763; 2000: 87 - 98.

24 Delahunty CM, Pigott JR, Conner JM and A Paterson Comparative volatile release from traditional and reduced fat cheddar cheeses upon mastication in the mouth. It. J. Food Sci. 1996; 2: 89 - 98. 